\title{
Superspace Collision: A Higher Dimensional Framework Describes Unexpected Supercell Refinement Results
}

\author{
Jeffrey J. Lovelace, Eppley Institute for Research in Cancer, Omaha, NE \\ Václav Petríček, Institute of Physics of the Czech Academy of Sciences, Prague, \\ Czech Republic \\ Garib N. Murshudov, MRC Laboratory of Molecular Biology, Cambridge, UK \\ Gloria E. O. Borgstahl, Eppley Institute for Research in Cancer, Omaha, NE
}

Protein crystals can be modulated ${ }^{1}$. Modulations come in two varieties: commensurate and incommensurate. Data with a commensurate modulation can be indexed and integrated using a supercell and refined using standard approaches. Incommensurate data must be handled using superspace methods $^{2}$. In superspace, for the 4D case, atoms are described as lines that follow a periodic path described by an atomic modulation function (AMF). The superspace approach is also valid for a commensurate modulation but the supercell pathway is much easier and currently the only option available leaving incommensurate data unsolvable for proteins at this time.

Previously, a commensurately modulated test dataset was created with both 3D and 4D representations ${ }^{3}$. A module to offload superspace calculations from REFMAC5 is under development to enable superspace refinement. In order to refine in superspace an initial guess at the AMFs is needed. One approach to get initial AMFs is to use a commensurate approximation and then perform traditional refinement and extract AMFs from the result. To test this approach an average starting structure was refined against the commensurate data. The refinement resulted in all of the AMFs out of phase by half a wavelength. This was confusing as the $3 \mathrm{D}$ space group $\mathrm{P} 212121$ has only one origin.

The superspace group $\mathrm{P} 212121(0 \beta 0)$ has two origin possibilities ( $\mathrm{x}_{4}$ and $\mathrm{x} 4+0.5$ ) or valid solutions. The $\mathrm{x}_{4}$ dimension contains the periodic AMFs. The AMFs describe a continuum of possible atomic states in 3D space. For commensurate structures only a small subset of these states are found in the crystal ( 7 in the test dataset). The $\mathrm{x}_{4}+0.5$ describes a slightly different set of states but these states are still describing the same AMFs. The end result is a slightly higher $\mathrm{R}$ $(0.1 \%)$ for the $\mathrm{x}_{4}+0.5$ (phase shifted) vs $\mathrm{x}_{4}$ (correct) refinement. The refinement starting point is equidistant from both solutions and the error well of the incorrect solution is shallower and wider resulting in the refinement funneling to the phase shifted solution.

1. Lovelace, JJ (2008) J. Appl. Cryst. 41:600-05.

2. Porta, JC (2011) J. Appl. Cryst. D67:628-38.

3. Lovelace, JJ (2013) Acta. Cryst. D69:1062-72. 